\title{
BODY SHAPE QUESTIONNAIRE (BSQ): ESTRUCTURA FACTORIAL Y FIABILIDAD EN UNIVERSITARIOS PERUANOS
}

\section{Body Shape Questionnaire (BSQ): Factorial structure and reliability in Peruvian university students}

\author{
Jonatan Baños-Chaparro* \\ Universidad Privada Norbert Wiener. Perú \\ Kassandra Aguilar Marca** \\ Universidad Alas Peruanas. Perú
}

\begin{abstract}
Resumen
La insatisfacción corporal es el descontento con la apariencia o el tamaño de algunas partes del cuerpo y se ha evidenciado alto niveles de prevalencia en población universitaria quienes constantemente se preocupan por su imagen física. Por ello, el estudio buscó analizar el proceso de validez basado en la estructura y consistencia interna del BSQ en una muestra de estudiantes universitarios. La Investigación es de tipo instrumental y 272 mujeres fueron evaluadas utilizando el BSQ. Mediante un análisis factorial confirmatorio se comprobó que el modelo unidimensional explicaba mejor el constructo a comparación de los modelos propuestos por otros estudios. Además, se evidenció excelente consistencia interna y de constructo mediante el coeficiente $\omega$ y $\mathrm{H}$. Los resultados apoyan el uso del BSQ en población universitaria y su estructura unidimensional. Además, se destaca la importancia de evaluar la insatisfacción corporal en estudiantes universitarios.
\end{abstract}

Palabras clave: insatisfacción corporal, análisis factorial, estudiantes universitarios

\begin{abstract}
Body dissatisfaction is disspleasure with the appearance or size of some parts of the body and high levels of prevalence have been evidenced in the university population that constantly worries about their physical image. Therefore, the study sought to analyze the validity process based on the structure and internal consistency of the BSQ in a sample of university students. The research is of type instrumental and 272 women were evaluated using the BSQ. By means of a confirmatory factor analysis checked that the one-dimensional model explained the construct better compared to the models proposed by other studies. Furthermore, evidenced excellent internal consistency and of construct by means of the $\omega$ and $H$ coefficients. The results support the use of the BSQ in the university population and its one-dimensional structure. Furthermore, the importance of evaluating body dissatisfaction in university students is highlighted.
\end{abstract}

Keywords: body dissatisfaction, factorial analysis, university students

Cualquier uso que se haga de este artículo debe incluir: Autor / Título original de la publicación / ISSN.
* Psicólogo.banos.jhc@gmail.com

Orcid: https://orcid.org/0000-0002-2604-7822

** Psicóloga. kassandraagm@gmail.com

Orcid: https://orcid.org/0000-0002-8691-0958 


\section{INTRODUCCIÓN}

La noción sobre la imagen corporal es variable debido a que está relacionado con la época y cultura (Gonçalves \& Bedin, 2016). En la actualidad, el contexto social propone que un cuerpo perfecto es sinónimo de un cuerpo delgado y ningún tipo de defecto, reforzado socialmente como una persona exitosa, agradable y bella (Amaya et al., 2010; TrejoOrtíz et al., 2016). Por ello, la cultura actual asume que alcanzar un cuerpo "ideal" es un logro significativo y de suma importancia, siendo los jóvenes los que presentan mayor preocupación por su apariencia física (Trejo-Ortíz et al., 2016). Sin embargo, jordanas extremas de ejercicio o dietas pueden ser un riesgo para la salud (Herraiz-Serrano et al., 2015) o no lograr tal objetivo ocasiona una preocupación constante por el peso o la apariencia, provocando una insatisfacción corporal (Trejo-Ortíz et al., 2016).

En ese sentido, la insatisfacción corporal es el disgusto con la apariencia o el tamaño de algunas partes del cuerpo (Acosta \& Gómez, 2003) y es un factor de riesgo considerable para los Trastornos de la Conducta Alimentaria (TCA) (Rohde, Stice \& Marti, 2015). Existen factores socioculturales que contribuyen a la insatisfacción corporal, como los medios de comunicación exponiendo modelos ideales (Moreno-Domínguez et al., 2019) o las presiones para adelgazar por parte de los familiares y amigos (Rohde et al., 2015). Aunado a esta situación, las diferencias individuales como el rasgo de personalidad perfeccionista (Holland et al., 2013), la baja autoestima (Berengüí et al., 2016) o altos niveles de neuroticismo (Allen \& Robson, 2020) son indicadores para predecir el desarrollo de un TCA.

Diversos estudios señalan a los estudiantes universitarios como una población vulnerable debido a la constante preocupación por su imagen física (Medina-Gómez et al., 2019; Trejo-Ortíz et al., 2016), evidenciando altos niveles de insatisfacción corporal (Benel et al., 2012; Berengüí et al., 2016). Por otro lado, un estudio epidemiológico realizado en el Perú, indicó que las mujeres de Lima y Callao se encontraban satisfechas con su apariencia física (69\%), aunque un $31 \%$ consideró lo opuesto. También se preguntó sobre los motivos de intento de suicidio y un mínimo porcentaje (1.9\%) señaló problemas con la autoimagen (Instituto Nacional de Salud Mental [INSM], 2013), siendo fundamental abordar esta problemática en las mujeres.

Una manera de poder hacerlo, es realizar la evaluación de la insatisfacción corporal mediante instrumentos psicométricos. En el contexto nacional se cuenta con evidencias de validez basadas en estructura y consistencia interna del Cuestionario de Insatisfacción con la Imagen Corporal (IMAGEN) (Hueda, 2018) y recientemente el Sociocultural Attitudes Toward Appearance Questionnaire 4 (SATAQ-4) (Zevallos-Delzo et al., 2020) que evalúa las presiones socioculturales que influyen en la insatisfacción corporal. Ambas validaciones en población universitaria.

No obstante, no se reporta evidencias de validez basadas en la estructura factorial y consistencia interna del Body Shape Questionnaire (BSQ) que evalúa la insatisfacción corporal y ha sido ampliamente analizado en Brasil (Da Silva et al., 2014), Colombia (Castrillón-Moreno et al., 2007), México (DominguezLara et al. 2018), Estados Unidos (Franko et al., 2012), España (Raich et al., 1996), Francia (Rousseau et al., 2005) y entre otros países europeos (Kling et al., 2019). La versión original ha demostrado una estructura unidimensional (Cooper et al., 1987), sin embargo, estudios posteriores han sugerido estructuras de dos factores (Castrillón-Moreno, et al., 2007), tres factores (Ochoa et al., 2007) y cuatro factores (Rousseau et al., 2005) considerando los 34 ítems o una versión de 18 ítems con cinco factores (Raich et al., 1996), siendo necesario analizar los modelos propuestos (tabla 1). 
Tabla 1

Modelos psicométricos del BSQ

\begin{tabular}{|c|c|c|}
\hline Modelo & Factores & Ítems \\
\hline $\mathrm{M}_{1}$ & F1: Insatisfacción corporal & F1: 1-34 \\
\hline $\mathrm{M}_{2}$ & $\begin{array}{l}\text { F1: Insatisfacción corporal } \\
\text { F2: Preocupación por el peso }\end{array}$ & $\begin{array}{l}\mathrm{F} 1: 1,3,7,8,10,11,12,13,14,15,17,18,19,20,25, \\
26,27,29,31,32 \\
\mathrm{~F} 2: 2,4,5,6,9,16,21,22,23,24,28,30,33,34\end{array}$ \\
\hline $\mathrm{M}_{3}$ & $\begin{array}{l}\text { F1: Insatisfacción corporal } \\
\text { F2: Molestias con la imagen corporal } \\
\text { F3: Devaluación de la figura }\end{array}$ & $\begin{array}{l}F 1: 7,9,10,12,13,14,15,17,18,19,20,25,29,31,23 \\
F 2: 1,2,4,5,6,21,22,24,28,34 \\
F 3: 3,8,11,16,26,27,30,32,33\end{array}$ \\
\hline $\mathrm{M}_{4}$ & $\begin{array}{l}\text { F1: Evitación y vergüenza social por } \\
\text { exposición del cuerpo } \\
\text { F2: Insatisfacción corporal con las partes } \\
\text { inferiores del cuerpo } \\
\text { F3: Uso de laxantes y vómitos } \\
\text { F4: Creencias y comportamientos } \\
\text { disfuncionales para controlar el peso }\end{array}$ & $\begin{array}{l}F 1: 7,8,15,18,19,20,27,31 \\
F 2: 2,3,4,5,9,10,12,14,24,25,28 \\
F 3: 11,26,32 \\
F 4: 6,21,22,23,34\end{array}$ \\
\hline $\mathrm{M}_{5}$ & $\begin{array}{l}\text { F1: Preocupación por el peso en relación } \\
\text { con la ingesta } \\
\text { F2: Aspectos desagradables de la obesidad } \\
\text { F3: Insatisfacción y preocupación por } \\
\text { el cuerpo general } \\
\text { F4: Insatisfacción corporal con la parte } \\
\text { inferior del cuerpo } \\
\text { F5: Uso de vómitos y uso de laxantes para } \\
\text { reducir la insatisfacción con el cuerpo }\end{array}$ & $\begin{array}{l}F 1: 2,6,14,17,19,21,23 \\
F 2: 5,16,28,30 \\
F 3: 7,13,18 \\
F 4: 3,10 \\
F 5: 26,32\end{array}$ \\
\hline
\end{tabular}

En ese sentido, la justificación teórica radica en poder identificar la estructura factorial del BSQ en base a la evidencia empírica propuesta por la literatura y validar sus puntaciones en población universitaria, a nivel práctico la evidencia de validez basada en la estructura y consistencia interna del BSQ permitirá ser utilizado en la investigación básica o aplicada, cuyos resultados podrán visibilizar el problema de la insatisfacción corporal ante la sociedad, comprendiendo su magnitud y consideración para acciones o políticas de salud pública.

\section{MÉTODO}

\section{Participantes}

Participaron 272 estudiantes de psicología de una universidad privada de Lima. Las estudiantes fueron seleccionadas mediante un muestreo no probabilístico por conveniencia y se encontraban matriculadas en el período 2018-1, turno mañana. Los criterios de inclusión fueron: estudiante matriculado de la escuela académico profesional de psicología, ser del sexo femenino y haber aceptado participar en el estudio. Los criterios de exclusión fueron: estudiantes no matriculados o retirados, ser del sexo masculino, no 
desear participar en la investigación y cuestionarios incompletos o con más de una respuesta. En ese sentido, la edad promedio de las participantes fue de 22 con una desviación estándar de \pm 4.28 en un rango de 17 a 39 años. Respecto al estado civil, el 93\% de las estudiantes se encontraba soltera y solo el $7 \%$ casada.

\section{Instrumento}

El BSQ desarrollado en Inglaterra (Cooper et al., 1987) y traducido al español por investigadores de España (Raich et al., 1996), es un instrumento unidimensional que evalúa la insatisfacción con la imagen corporal mediante 34 ítems y su sistema de respuesta es de seis opciones: que va desde 1 punto equivalente a la categoría nunca hasta 6 puntos equivalente a la categoría siempre. En este estudio se utilizó la versión en español.

\section{Procedimiento}

En principio, se realizó un estudio piloto con 15 estudiantes de psicología, con el objetivo de analizar la claridad de los ítems. En ese sentido, se reemplazó la palabra «carne» por «cuerpo» de los ítems 5, 8 y 28. Además, se modificó la redacción del ítem 29 "verte reflejada en un espejo o en un escaparate, ¿te ha hecho sentir mal por tu figura?" por "verte reflejada en un espejo, ite ha hecho sentir mal por tu figura?". Posteriormente se procedió a la aplicación de forma colectiva, donde las estudiantes que aceptaron participar voluntariamente y firmaron el asentimiento informado se les explicó las instrucciones para resolver el instrumento. Finalmente, la información fue digitada y revisada en una base de datos de Excel con la finalidad de evitar cualquier error de digitación.

\section{Análisis de datos}

Inicialmente se realizó el análisis descriptivo de los ítems, considerando los estadísticos de distribución (media y desviación estándar), asimetría (g1) y curtosis (g2), con el objetivo de observar el comportamiento de los indicadores.

Respecto al análisis factorial confirmatorio (AFC), se utilizó el estimador robusto de mínimos cuadrados ponderados ajustados a la media y la varianza (WLSMV) recomendado para ítems ordinales (Kline, 2016) y los índices de bondad de ajuste fueron los siguientes: índice de ajuste comparativo (CFI), índice de Tucker-Lewis (TLI), raíz normalizada cuadrada media residual (SRMR) y el error medio cuadrático de aproximación (RMSEA) reportado con sus intervalos de confianza (IC) al 90\%. Para que el ajuste sea adecuado, los valores de CFI y TLI deberán ser superior a 0.90 (Bentler, 1990), los valores de SRMR menor a 0.08 y RMSEA menor a 0.06 (Hu \& Bentler, 1999).

Por otro lado, el análisis de consistencia interna se estimó con el coeficiente omega $(\omega)$, cuyo valor deberá ser superior a 0.70 y reportado con IC al $95 \%$ en mil muestras bootstrap (Ventura-León, 2017) y la confiabilidad de constructo mediante el coeficiente $\mathrm{H}$, siendo óptimo un valor superior a 0.70 (DomínguezLara, 2016).

Los análisis estadísticos se realizaron en el software $\mathrm{R}$ versión 3.6.2 (R Core Team, 2019) utilizando el paquete Lavaan (Rosseel, 2012), semPlot (Epskamp, 2015) y MBESS (Kelley, 2007). Para el cálculo del coeficiente $\mathrm{H}$ se utilizó el software FACTOR versión 10.10.02 (Lorenzo-Seva \& Ferrando, 2006).

\section{RESULTADOS}

La tabla 1 indica que el rango de la media se encuentra entre 0.46 y 2.34 , es decir, entre las preguntas: "¿has vomitado para sentirte más delgada?" y "la preocupación por tu figura ¿te ha hecho pensar que deberías hacer ejercicio?". Asimismo, la desviación menor se encuentra en el ítem 11 y la mayor en el ítem 4. En relación a la asimetría y curtosis, a excepción de algunos ítems, la mayoría se encuentra entre \pm 2.00 (George \& Mallery, 2016). Por último, se evidencia aceptables cargas factoriales $(\lambda)$, los cuales fueron superior a 0.50 (Lloret, Ferreres, Hernández \& Tomás, 2017). 
Tabla 2

Estadísticos descriptivos de la escala BSQ

\begin{tabular}{|c|c|c|c|c|c|}
\hline & Media & DE & g1 & g2 & $\lambda$ \\
\hline Ítem 1 & 1.39 & 1.35 & 0.97 & 0.45 & 0.582 \\
\hline Ítem 2 & 2.19 & 1.44 & 0.41 & -0.52 & 0.739 \\
\hline Ítem 3 & 1.14 & 1.44 & 1.23 & 0.68 & 0.507 \\
\hline Ítem 4 & 2.26 & 1.63 & 0.30 & -1.03 & 0.726 \\
\hline Ítem 5 & 2.00 & 1.44 & 0.48 & -0.57 & 0.597 \\
\hline Ítem 6 & 1.73 & 1.59 & 0.60 & -0.74 & 0.750 \\
\hline Ítem 7 & 0.63 & 1.16 & 2.11 & 4.15 & 0.663 \\
\hline Ítem 8 & 0.61 & 1.11 & 1.99 & 3.45 & 0.649 \\
\hline Ítem 9 & 1.13 & 1.41 & 1.28 & 0.92 & 0.715 \\
\hline Ítem 10 & 0.83 & 1.24 & 1.76 & 2.83 & 0.626 \\
\hline Ítem 11 & 0.55 & 0.96 & 2.00 & 4.26 & 0.653 \\
\hline Ítem 12 & 1.61 & 1.31 & 0.86 & 0.45 & 0.683 \\
\hline Ítem 13 & 0.70 & 1.12 & 2.08 & 4.37 & 0.635 \\
\hline Ítem 14 & 1.14 & 1.24 & 1.21 & 1.27 & 0.690 \\
\hline Ítem 15 & 1.54 & 1.47 & 0.86 & -0.09 & 0.598 \\
\hline Ítem 16 & 1.78 & 1.51 & 0.68 & -0.37 & 0.709 \\
\hline Ítem 17 & 1.27 & 1.44 & 1.08 & 0.37 & 0.773 \\
\hline Ítem 18 & 0.59 & 1.03 & 2.13 & 5.00 & 0.708 \\
\hline Ítem 19 & 0.97 & 1.30 & 1.39 & 1.28 & 0.784 \\
\hline Ítem 20 & 1.29 & 1.32 & 0.94 & 0.33 & 0.689 \\
\hline Ítem 21 & 1.61 & 1.51 & 0.72 & -0.35 & 0.755 \\
\hline Ítem 22 & 1.47 & 1.55 & 0.90 & -0.22 & 0.696 \\
\hline Ítem 23 & 1.58 & 1.58 & 0.73 & -0.54 & 0.760 \\
\hline Ítem 24 & 1.70 & 1.47 & 0.66 & -0.33 & 0.687 \\
\hline Ítem 25 & 0.96 & 1.40 & 1.46 & 1.14 & 0.661 \\
\hline Ítem 26 & 0.46 & 1.02 & 2.65 & 7.43 & 0.564 \\
\hline Ítem 27 & 0.60 & 1.09 & 2.32 & 5.69 & 0.696 \\
\hline Ítem 28 & 1.32 & 1.38 & 1.00 & 0.37 & 0.572 \\
\hline Ítem 29 & 1.22 & 1.32 & 1.14 & 0.74 & 0.754 \\
\hline Ítem 30 & 1.32 & 1.41 & 0.99 & 0.27 & 0.657 \\
\hline Ítem 31 & 1.31 & 1.37 & 0.94 & 0.21 & 0.668 \\
\hline Ítem 32 & 0.63 & 1.18 & 2.03 & 3.64 & 0.657 \\
\hline Ítem 33 & 1.37 & 1.31 & 0.95 & 0.49 & 0.735 \\
\hline Ítem 34 & 2.34 & 1.58 & 0.27 & -0.96 & 0.718 \\
\hline
\end{tabular}

Nota. DE: desviación estándar, g1: asimetría, g2: curtosis, $\lambda$ : carga factorial. 
En cuanto al AFC de los modelos reportados por estudios psicométricos, se evidencia que todos los modelos (M1, M2, M3, M4 y M5) presentan adecuados índices de ajuste. Sin embargo, la correlación interfactorial del modelo de dos $(\psi=$
$0.94)$, tres $(\psi$ promedio $=0.94)$, cuatro $(\psi$ promedio $=0.88)$ y cinco factores $(\psi$ promedio $=0.77)$ fue alta, indicando la presencia de un factor general. En ese sentido, el modelo unidimensional es el que explica mejor el constructo.

Tabla 3

Índices de ajuste de los modelos del BSQ

\begin{tabular}{lccccccc}
\hline & $\boldsymbol{\chi 2}$ & $\mathbf{g l}$ & $\boldsymbol{\chi} \mathbf{2} / \mathbf{g l}$ & $\mathbf{C F I}$ & TLI & SRMR & RMSEA [IC 90\%] \\
\hline $\mathrm{M}_{1}$ & $981.155^{*}$ & 527 & 1.86 & 0.950 & 0.947 & 0.061 & $0.056[0.051-0.062]$ \\
$\mathrm{M}_{2}$ & $948.608^{*}$ & 526 & 1.80 & 0.953 & 0.950 & 0.060 & $0.054[0.049-0.060]$ \\
$\mathrm{M}_{3}$ & $943.450^{*}$ & 524 & 1.80 & 0.954 & 0.951 & 0.060 & $0.054[0.049-0.060]$ \\
$\mathrm{M}_{4}$ & $619.157^{*}$ & 318 & 1.94 & 0.955 & 0.950 & 0.059 & $0.059[0.052-0.066]$ \\
$\mathrm{M}_{5}$ & $234.302^{*}$ & 125 & 1.87 & 0.974 & 0.968 & 0.047 & $0.057[0.045-0.068]$ \\
\hline
\end{tabular}

Nota. ${ }^{*} \mathrm{p}<0.00 ; \mathrm{M}_{1}=$ un factor; $\mathrm{M}_{2}=$ dos factores; $\mathrm{M}_{3}=$ tres factores, $\mathrm{M}_{4}=$ cuatro factores, $\mathrm{M}_{5}=$ cinco factores.

\section{Figura 1}

Estructura factorial del Body Shape Questionnaire

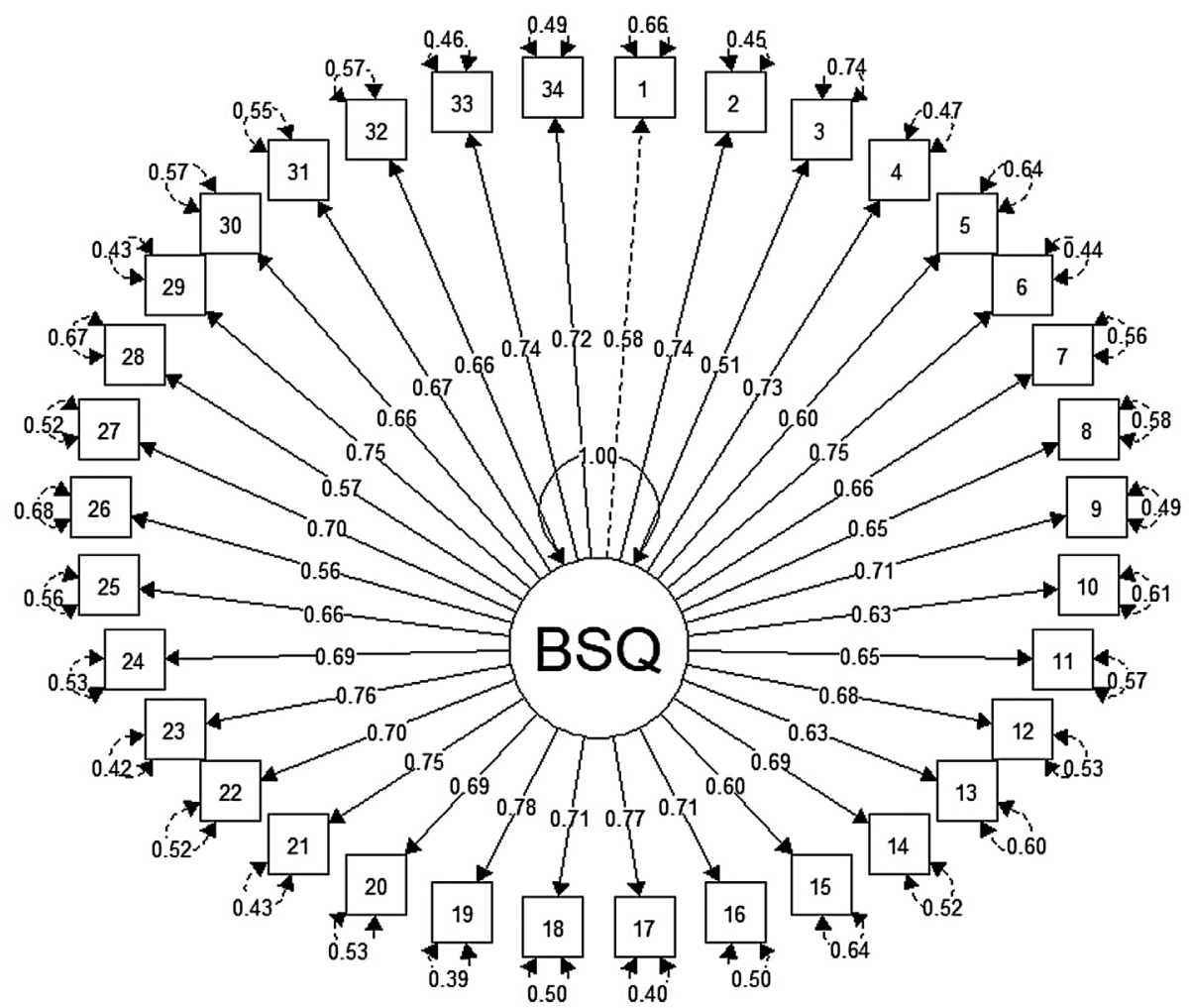


Por último, respecto al análisis de la consistencia interna, se obtuvo valores óptimos del coeficiente $\omega=0.96$ [0.94-0.96]. Además, se reportan valores adecuados de confiabilidad del constructo H-Latente $=0.96[0.95-0.97] \mathrm{y}$ de las puntuaciones observadas $\mathrm{H}$-Observada $=0.88$ [0.86-0.89].

\section{DISCUSIÓN}

La insatisfacción corporal es un factor de riesgo asociado al TCA (Rohde et al., 2015) y con mayor prevalencia en las mujeres (Acosta \& Gómez, 2003; Amaya et al., 2010). Se ha evidenciado que este problema se encuentra presente en estudiantes universitarios con altos niveles de insatisfacción (Benel et al., 2012; Medina-Gómez et al., 2019), lo que podría generar un problema de salud sino se realiza una intervención anticipada. Para ello, es necesario disponer de herramientas con evidencias de validez en población universitaria para una evaluación más precisa y confiable. En ese sentido, el objetivo del estudio fue realizar la validez basada en la estructura y consistencia interna del BSQ.

Para tal objetivo, se llevó a cabo un AFC donde se probaron cinco modelos recopilados de la evidencia empírica. En ese sentido, el modelo de un factor (M1) explicó mejor el constructo a comparación de un modelo de dos (M2), tres (M3), cuatro (M4) o cinco factores (M5) debido a la alta correlación interfactorial que presentaban. Esto no es una sorpresa cuando se revisa otras investigaciones que reportan altas correlaciones de factores evidenciando multicolinealidad, es decir, ausencia de independencia de cada factor (Da Silva, Marôco \& Campos, 2018; Dominguez-Lara et al., 2018) afirmando la presencia de un factor general que representa apropiadamente la estructura interna y que no significa un conjunto espurio de ítems. En ese sentido, los resultados de la investigación concuerdan con otros estudios del BSQ como la evidencia de validez en Brasil (Da Silva et al., 2014), México (Dominguez-Lara et al., 2018), Estados Unidos (Franko et al., 2012) y otros análisis psicométricos en muestras europeas (Kling et al., 2019).

En ese sentido, la insatisfacción corporal es comprendida como un constructo unidimensional que explica la preocupación del individuo por el tamaño y forma de algunas partes de su cuerpo, respaldada teóricamente por la dimensión actitudinal de la imagen corporal (Cooper et al., 1987) y que no existe justificación teórica para considerar un modelo multidimensional (Da Silva et al., 2018). Los estudios con evidencia de más de un factor (Castrillón-Moreno et al., 2007; Ochoa et al., 2007; Raich et al., 1996; Rousseau et al., 2005), no presentan sustento teórico para la estructura de estos factores y en algunos casos se utiliza el análisis factorial exploratorio, el cual no es recomendable cuando ya existe una teoría desarrollada para un instrumento (Da Silva et al., 2018).

Respecto a la consistencia interna mediante el coeficiente $\omega$ basado en las cargas factoriales, se evidenció valores óptimos, lo que permite concluir que el contenido de los ítems es homogéneo y explican un mismo constructo. Además, el índice de confiabilidad de constructo mediante el coeficiente $\mathrm{H}$ demostró valores excelentes, lo cual indica que la variabilidad del constructo se encuentra explicada por lo ítems y que es coherente y rigurosa (Domínguez-Lara, 2016).

No obstante, la investigación presenta varias limitaciones, por ejemplo, una importante limitación del estudio fue la participación de estudiantes universitarios lo que no permite generalizar los resultados en niños, estudiantes de educación básica o muestras clínicas, siendo necesario estudios confirmatorios en aquellas poblaciones. Otra limitación se encuentra vinculada a la evidencia de validez convergente o divergente que brindaría mayor validez a la estructura factorial con otros constructos similares u opuestos, también el análisis de invarianza factorial que apoyaría la igualdad o diferencia de los grupos evaluados.

En conclusión, los resultados de la investigación respaldan el modelo teórico original unidimensional (Cooper et al., 1987) y las puntuaciones derivadas del BSQ en universitarios peruanos, lo que permite su uso en dicha población. En ese sentido, este estudio destaca la importancia de la evaluación de la insatisfacción corporal, mayor aún en universitarios donde se ha evidenciado la presencia de este problema. 


\section{REFERENCIAS}

Acosta, M., \& Gómez, G. (2003). Insatisfacción corporal y seguimiento de dieta. Una comparación transcultural entre adolescentes de España y México. International Journal of Clinical and Health Psychology, 3(1), 9-21. https://www. redalyc.org/articulo.oa?id=33730101

Allen, M. S., \& Robson, D. A. (2020). Personality and body dissatisfaction: An updated systematic review with meta-analysis. Body Image, 33, 77-89. https://doi.org/doi.org/10.1016/j. bodyim.2020.02.001

Amaya, A., Álvarez, G., \& Mancilla, J. (2010). Insatisfacción corporal en interacción con autoestima, influencia de pares y dieta restrictiva: Una revisión. Revista Mexicana de Trastosnos Alimentarios, 1(1), 76-89. http://www.scielo. org. $\mathrm{mx} / \mathrm{scielo}$.php?script=sci_arttext\&pid $=\mathrm{S} 2007-15232010000100008$

Benel Huerta, R., Campos Campos, S., \& Cruzado, L. (2012). Insatisfacción corporal en estudiantes de medicina de la Universidad Peruana Cayetano Heredia medida con el Body Shape Questionnaire. Revista de Neuro-Psiquiatria, 75(3), 85-92. https://doi.org/10.20453/rnp.v75i3.1226

Bentler, P. M. (1990). Comparative fit indexes in structural models. Psychological bulletin, 107(2), 238-246. https://doi.org/10.1037/00332909.107.2.238

Berengüí, R., Castejón, M. A., \& Torregrosa, M. S. (2016). Body dissatisfaction, risk behaviors eating disorders in university students. Revista Mexicana de Trastornos Alimentarios, 7, 1-8. https://doi.org/10.1016/j.rmta.2016.02.004

Castrillón-Moreno, D., Luna-Montaño, I., AvendañoPrieto,G.,\&Pérez-Acosta,A.M.(2007).Validación del Body Shape Questionnaire (Cuestionario de la figura corporal) BSQ para la población colombiana. Act Colomb Psicol, 10, 15-23. http:// www.scielo.org.co/scielo.php?script=sci_arttext \&pid=S0123-91552007000100003

Cooper, P. J., Taylor, M. J., Cooper, Z., \& Fairburn, C. G. (1987). The development and validation of the Body Shape Questionnaire. Int J Eat Disord,
6(4), 485-494. https://doi.org/10.1002/1098108 X ( 198707 ) 6:4\% 3 c 485 : A I D EAT2260060405\%3e3.0.CO;2-O

Da Silva, W. R., Dias, J. C., Marôco, J., \& Campos, J. A. (2014). Confirmatory factor analysis of different versions of the Body Shape Questionnaire applied to Brazilian university students. Body Image, 11(4), 384-390. https://doi.org/10.1016/j. bodyim.2014.06.001

Da Silva, W. R., Marôco, J., \& Campos, J. A. (2018). Avaliação da estrutura fatorial do Body Shape Questionnaire: análise fatorial exploratória ou confirmatória? Jornal Brasileiro de Psiquiatria, 67(3), 201-207. https://doi.org/10.1590/00472085000000205

Domínguez-Lara, S. (2016). Evaluación de la confiabilidad del constructo mediante el coeficiente $\mathrm{H}$ : breve revisión conceptual y aplicaciones. Psychologia: Avances de la Disciplina, 10(2), 8794. https://doi.org/10.21500/19002386.2134

Dominguez-Lara, S., Aguirre-Pérez, S., RomoGonzález, T., Herrera-Meza, S., \& CamposUscanga, Y. (2018). Análisis psicométrico del Body Shape Questionnaire en universitarios mexicanos. Rev Colomb Psiquiat. https://doi. org/10.1016/j.rcp.2018.09.002

Epskamp, S. (2015). semPlot: Unified visualizations of structural equation models. Structural Equation Modeling, 22(3), 474-83. https://doi.org/10.1080/ 10705511.2014.937847

Franko, D. L., Jenkins, A., Roehrig, J. P., Luce, K. H., Crowther, J. H., \& Rodgers, R. F. (2012). Psychometric properties of measures of eating disorder risk in latina college women. International Journal of Eating Disorders, 45(4), 592-596. https://doi.org/10.1002/eat.20979

George, D., \& Mallery, P. (2016). IBM SPSS Statistics 23 Step by Step: A Simple Guide and Reference. Routledge.

Gonçalves, S., \& Bedin, L. (2016). Bienestar, salud e imagen corporal de adolescentes brasileros: la importancia de los contextos familiares, de amistad y escolar. Universitas Psychologica, 14(4), 1399-1410. https://doi.org/10.11144/ Javeriana.up14-4.bsica 
Herraiz-Serrano, C., Rodríguez-Cano, T., BeatoFernández, L., Latorre-Prostigo, J. M., RojoMoreno, L., \& Vaz-Leal, F. J. (2015). Parental rearing and eating psychopathology. Actas Españolas de Psiquiatría, 43(3), 91-98. https:// www.actaspsiquiatria.es/repositorio/17/95/ ENG/17-95-ENG-91-8-429121.pdf

Holland, L. A., Bodell, L. P., \& Keel, P. K. (2013). Psychological factors predict eating disorder onset and main-tenance at 10-year follow-up. European eating disorders review: the journal of the Eating Disorders Association, 21(5), 405-410. https://doi.org/10.1002/erv.2241

Hu, L. T., \& Bentler, P. M. (1999). Cutoff Criteria for Fit Indexes in Covariance Structure Analysis: Conventional Criteria Versus New Alternatives. Structural Equation Modeling, 6(1), 1-55. https:// doi.org/10.1080/10705519909540118

Hueda Capristán, A. C. (2018). Propiedades psicométricas del Cuestionario de Insatisfacción con la Imagen Corporal en universitarios. Revista de psicología, 19(1), 62-88. https://doi. org/10.18050/revpsi.v1i19.1267

Instituto Nacional de Salud Mental. (2013). Estudio Epidemiologico de Salud Mental en Lima Metropolitana y Callao Replicacion 2012. http://www.insm.gob.pe/investigacion/archivos/ estudios/2012\%20ASM\%20-EESM\%20-LM.pdf

Kelley, K. (2007). Methods for the Behavioral, Educational, and Social Sciences: An R package. Behavior Research Methods, 39, 979-84. https:// doi.org/10.3758/BF03192993

Kline, R. B. (2016). Principles and practice of structural equation modeling (Fourth edition). Methodology in the social sciences. New York: The Guilford Press.

Kling, J., Kwakkenbos, L., Diedrichs, P. C., Rumsey, N., Frisén, A., Brandão, M. P., . . . Fitzgerald, A. (2019). Systematic review of body image measures. Body Image, 30, 170-211. https://doi. org/10.1016/j.bodyim.2019.06.006

Lloret, S., Ferreres, A., Hernández, A., \& Tomás, I. (2017). The exploratory factor analysis of items: guided analysis based on empirical data and software. Anales de psicología, 33(2), 417-432. https://doi.org/10.6018/analesps.33.2.270211

Lorenzo-Seva, U., \& Ferrando, P. (2006). FACTOR: A computer program to fit the exploratory factor analysis model. Behavioral Research Methods, Instruments and Computers, 38(1), 88-91. https:// doi.org/10.3758/bf03192753

Medina-Gómez, M. B., Martínez-Martín, M. A., Escolar-Llamazares, M. C., González-Alonso, Y., \& Mercado-Val, E. (2019). Ansiedad e insatisfacción corporal en universitarios. Acta Colombiana de Psicología, 22(1), 13-21. https:// doi.org/10.14718/ACP.2019.22.1.2

Moreno-Domínguez, S., Servián-Franco, F., Reyes del Paso, G. A., \& Cepeda-Benito, A. (2019). Images of Thin and Plus-Size Models Produce Opposite Effects on Women's Body Image, Body Dissatisfaction, and Anxiety. Sex Roles, 80, 607616. https://doi.org/10.1007/s11199-018-0951-3

Ochoa, S., Villareal, L., Molina, N., \& Bayle, J. (2007). Validación y confiabilidad del Body Shape Questionnaire (BSQ) en adolescentes de la ciudad de colima. Revista científica electrónica de psicología, 4, 204-216. http://dgsa.uaeh.edu.mx/ revistas/index.php/psicologia/article/view/78/71

$\mathrm{R}$ Core Team. (2019). $R$ : A language and environment for statistical computing (version 3.6.2) [software]. R Foundation for Statistical Computing.

Raich, R. M., Mora, M., Soler, A., Avila, C., Clos, I., \& Zapater, L. (1996). Adaptación de un instrumento de evaluación de la insatisfacción corporal. Clínica y Salud, 7, 51-66. https://journals.copmadrid.org/ clysa/art/f2217062e9a397a1dca429e7d70bc6ca

Rohde,P.,Stice,E.,\& Marti,C.N.(2015).Development and predictive effects of eating disorder risk factors during adolescence: Implications for prevention efforts. International Journal of Eating Disorders, 48(2), 187-198. doi:10.1002/eat.22270

Rosseel, Y. (2012). lavaan: An R Package for Structural Equation Modeling. Journal of statistical software, 48(2), 36. https://doi.org/10.18637/jss. v048.i02

Rousseau, A., Knotter, A., Barbe, P., Raich, R. M., \& Chabrol, H. (2005). Étude de validation de la 
version française du Body Shapre Questionnaire. L'Encépchale,31,162-73.https://doi.org/10.1016/ S0013-7006(05)82383-8

Trejo-Ortíz, P. M., Mollinedo Montaño, F. E., Araujo Espino, R., Valdez-Esparza, G., \& Sánchez Bonilla, M. (2016). Hábitos de actividad física y cánones de imagen corporal en estudiantes universitarios. Revista Cubana de Medicina General Integral, 32(1), 72-82. http://scielo. sld.cu/scielo.php?script=sci_arttext\&pid $=$ S0864-21252016000100010
Ventura-León, J. (2017). Intervalos de confianza para coeficiente omega: propuesta para el cálculo. Adicciones, 30(1), 77-78. https://doi. org/10.20882/adicciones.962

Zevallos-Delzo, C., Maguiña, J. L., Catacora, M., \& Mayta-Tristán, P. (2020). Adaptación cultural y validación del SATAQ-4 "Sociocultural Attitudes towards appearance Questionnaire-4" para población peruana. REV. CHIL. NEUROPSIQUIAT, 58(1), 16-28. https://doi.org/10.4067/ S0717-92272020000100016

Fecha de recepción: 8 de octubre de 2020

Fecha de aceptación: 6 de noviembre de 2020 